Revue des patrimoines

Le patrimoine religieuX des $\mathrm{XIX} \mathrm{X}^{\mathrm{e}}$ et $\mathrm{XX} \mathrm{X}^{\mathrm{e}}$ siècles

\title{
Histoire, patrimoine immatériel et identité : la question religieuse au Québec
}

Sylvie Grenet

\section{(2) OpenEdition}

Journals

Édition électronique

URL : http://journals.openedition.org/insitu/4548

DOI : 10.4000/insitu.4548

ISSN : $1630-7305$

Éditeur

Ministère de la culture

Référence électronique

Sylvie Grenet, « Histoire, patrimoine immatériel et identité : la question religieuse au Québec », In Situ [En ligne], 11 | 2009, mis en ligne le 18 avril 2012, consulté le 19 avril 2019. URL : http:// journals.openedition.org/insitu/4548; DOI : 10.4000/insitu.4548

Ce document a été généré automatiquement le 19 avril 2019

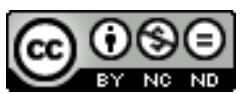

In Situ Revues des patrimoines est mis à disposition selon les termes de la licence Creative Commons Attribution - Pas d'Utilisation Commerciale - Pas de Modification 4.0 International. 


\title{
Histoire, patrimoine immatériel et identité : la question religieuse au Québec
}

\author{
Sylvie Grenet
}

1 En guise d'introduction, nous avons choisi de nous attarder sur un débat qui nous semble résumer les problèmes liés aux questions du patrimoine religieux au Québec. Pour bien le comprendre, il est nécessaire de fournir quelques indications sur le contexte dans lequel il a surgi, à savoir, celui des « accommodements raisonnables ». Pour résumer, ce terme désigne une série de compromis qui ont permis aux différentes composantes de la nation canadienne (et pas seulement québécoise) de «vivre ensemble » depuis les années 1980. Issue de la jurisprudence du monde du travail, l'expression désigne « l'assouplissement d'une norme afin de contrer la discrimination que peut créer cette norme et que subit une personne, dans le but de respecter le droit à l'égalité du citoyen $»^{1}$. Il ne s'agit pas d'annuler une loi ou une disposition, mais d'en atténuer les effets potentiellement discriminatoires pour certaines personnes ou certains groupes. Ces compromis ont souvent porté sur les domaines religieux. Le premier cas remonte à 1985, date à laquelle la commission ontarienne des droits de la personne autorise un membre de l'église adventiste du Septième Jour à ne pas travailler le samedi matin. L'un des plus récents date de janvier 2008, lorsque la commission scolaire de Toronto s'engage à ouvrir en septembre 2009 une école offrant des enseignements consacrés à la culture afrocanadienne, suite à la demande de la communauté noire de Toronto. Depuis ces dernières années, les cas se sont multipliés, et tout particulièrement entre 2006 et 2007, où l'on recense $55 \%$ des cas. Souvent relayés par la presse, ces compromis font l'objet de très vifs débats, qui concernent d'ailleurs plus les éditorialistes ou les hommes politiques que les citoyens eux-mêmes². 
Figure 1

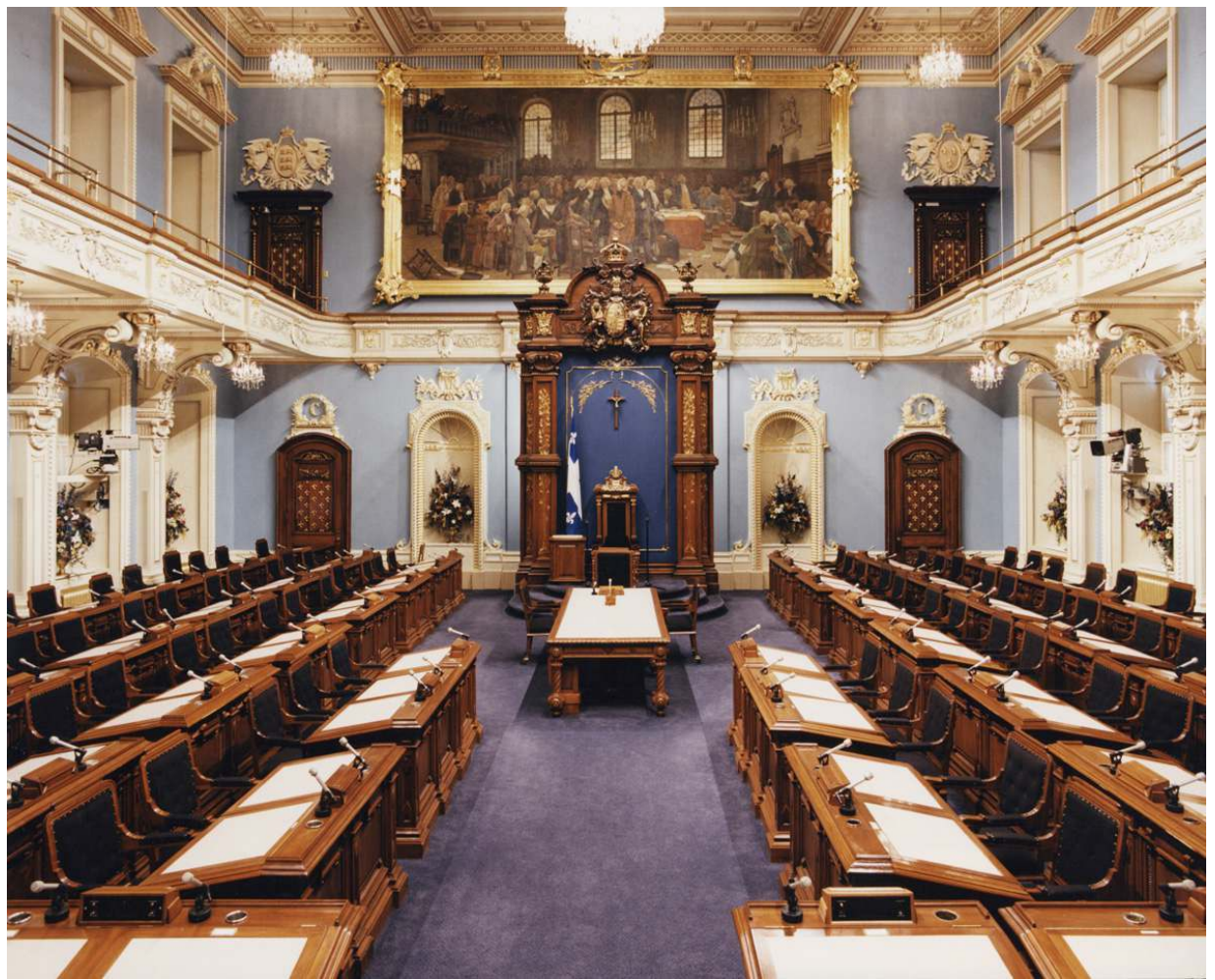

Vue de la salle des débats « salon bleu » de l'Assemblée Nationale du Québec (c) Collection de l'Assemblée Nationale du Québec

Figure 2

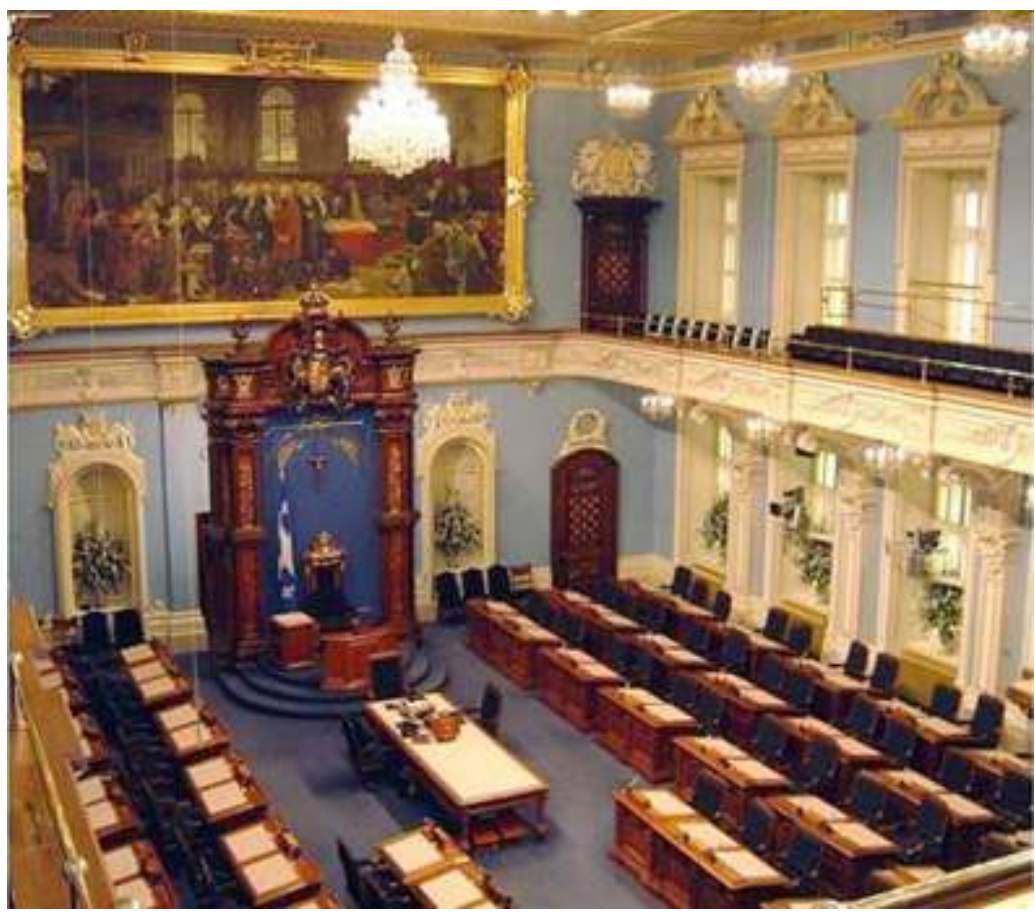

Vue de la salle des débats « salon bleu » de l'Assemblée Nationale du Québec (c) Collection de l'Assemblée Nationale du Québec 
Pour mieux analyser la situation au Québec, le gouvernement québécois a demandé à deux universitaires, Charles Taylor ${ }^{3}$ et Gérard Bouchard ${ }^{4}$, de présider une commission chargée d'étudier " les pratiques d'accommodements reliées aux différences culturelles ${ }^{5}$ . Le rapport de la commission a été rendu en mai 2008. Dans ce rapport de 310 pages, intitulé Fonder l'avenir. Le temps de la conciliation ${ }^{6}$, les auteurs affirment le principe de laïcité, inscrit dans la constitution du Québec.

Figure 3

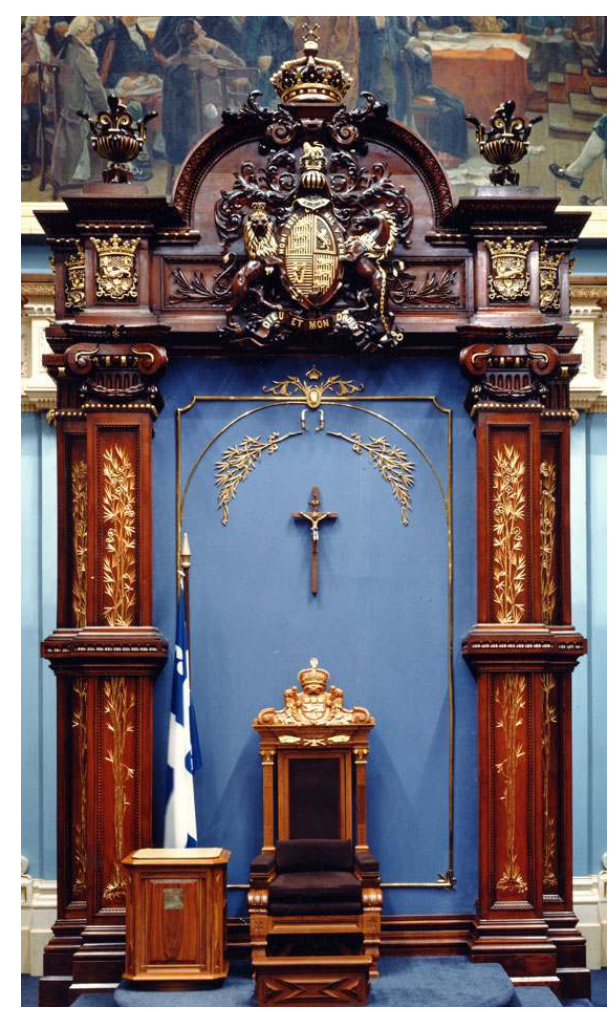

Siège du président de l'Assemblée Nationale du Québec

(c) Collection de l'Assemblée Nationale du Québec

Conformément à ce principe, ils suggèrent, dans leurs recommandations prioritaires, d'enlever le crucifix accroché au-dessus du siège du président de l'Assemblée nationale depuis 1936 : «Au nom de la séparation de l'État et des Églises et de la neutralité de l'État, nous pensons qu'il faudrait enlever le crucifix accroché au mur de l'Assemblée nationale. Il s'agit là, en effet, du lieu même qui symbolise l'État de droit (une solution raisonnable serait de l'exposer dans une salle consacrée à l'histoire du Parlement) ${ }^{7}$.» (fig. $\mathbf{n}^{\circ}$ 3) Les auteurs proposent ainsi d'opérer un déplacement d'un symbole religieux, lié à l'exercice effectif du pouvoir politique, vers un espace lié au patrimoine historique de la nation.

La réponse du commanditaire face à cette tentative de neutralisation par le recours à l'histoire et au patrimoine, ne s'est pas fait attendre. Dans les minutes qui ont suivi le dépôt du rapport, le gouvernement du Québec a voté à l'unanimité une motion stipulant que: «L'Assemblée nationale réitère sa volonté de promouvoir la langue, l'histoire, la culture et les valeurs de la nation québécoise, favorise l'intégration de chacun à notre nation dans un esprit d'ouverture et de réciprocité et témoigne de son attachement à notre patrimoine religieux et historique représenté par le crucifix de notre Salon bleu et nos armoiries ornant nos institutions ${ }^{8}$. » Dans la conférence de presse qui a suivi le dépôt 
de cette motion, le président de l'assemblée, Jean Charest, a de nouveau insisté sur l'importance de conserver le crucifix dans l'enceinte politique : "C'est notre histoire, on ne peut écrire l'histoire à l'envers. L'Église a joué un rôle important dans l'histoire du Québec et le crucifix est le symbole de cette histoire ${ }^{9}$.» Dans les deux cas, c'est la dimension historique et patrimoniale du symbole religieux qui est en question, mais alors que Charles Taylor et Gérard Bouchard invoquent un retrait du patrimoine religieux, pour un lieu qui serait dénué de charge politique, Jean Charest réintroduit le politique au sein du patrimoine historique religieux.

5 Cet épisode nous semble révélateur des oscillations que nous avons pu constater pendant notre mission sur place ${ }^{10}$, entre mai et juin 2008, entre histoire, patrimonialisation et reconnaissance identitaire, lorsqu'il s'agit d'aborder la question religieuse au Québec. Notre but est de tenter de rendre compte, sous cet angle, de la manière dont est en train de se définir le patrimoine religieux au Québec, en étudiant le rôle des administrations culturelles, et tout particulièrement du ministère de la Culture québécois. Après un survol rapide de la situation des religions au Québec, nous aborderons ensuite plus précisément les mesures mises en place par le ministère de la Culture québécois pour identifier et faire connaître le patrimoine religieux, tout particulièrement immatériel, de la province.

\section{La religion au Québec : de l'unicité à la diversité}

Dans Genèse de la société québécoise, le sociologue Fernand Dumont évoque son enfance d'avant la Seconde Guerre mondiale, vécue dans les limites réconfortantes d'une ville, Montmorency, qui se définissait avant tout comme une communauté soudée, organiquement liée à la religion catholique, se confondant volontiers avec la paroisse : «Parmi ceux de ma génération, beaucoup ont été des enfants de paroisse. [...] La religion du Québec d'antan s'engrenait si bien aux rythmes des jours et des saisons qu'elle semblait enlisée dans l'ici-bas ${ }^{11}$.»

7 Le fait est que la religion catholique a joué un rôle primordial dans le tissu politique, social, intellectuel et artistique du Québec, forgeant une identité dont le Québec d'aujourd'hui lui est largement redevable. Dans les années 1960 pourtant, s'amorce un déclin de la prégnance catholique, dans la mouvance de la "Révolution tranquille», terme utilisé pour désigner une évolution profonde de la société québécoise sur les plans politique, social, culturel et religieux. On constate une baisse spectaculaire du nombre de catholiques pratiquants, qui passent en 30 ans, de $80 \%$ dans les années 1970, à moins de $5 \%$ en $2000^{12}$. Ce mouvement s'est accompagné d'une baisse du recrutement régulier, et $\mathrm{du}$ vieillissement des membres des congrégations religieuses. Luc Noppen et Lucie K. Morisset estimaient dans un ouvrage paru en $2005{ }^{13}$ qu'en 2007, il n'y aurait plus environ que 60 prêtres pour desservir 233 paroisses, soit un prêtre pour trois paroisses en moyenne.

8 Alors que la religion catholique perd ses pratiquants et voit ses bâtiments fermer, le Québec doit faire face à l'émergence d'autres religions, qu'elles soient le produit de la résurgence de pratiques autochtones, ou qu'elles émanent de populations récemment immigrées ${ }^{14}$. Selon un recensement de l'Institut de la statistique du Québec mené sur les religions des Québécois en 2001, sur une population d'environ 7 millions d'habitants, 6 millions se déclaraient catholiques, 330000 protestants, 108000 musulmans, 100000 orthodoxes, 90000 juifs, 40000 se déclaraient sans croyance, 140000 pratiquant 
d'«autres» religions (dont, par ordre numérique décroissant, 50000 bouddhistes, 35000 Témoins de Jéhovah, 30000 hindous et 8700 sikhs) ${ }^{15}$.

9 Le recensement souligne en outre l'importante proportion de migrants dans les « autres religions", soit environ $70 \%$ d'immigrants pratiquant les religions musulmanes, bouddhistes et hindoues contre seulement 5,5\% d'immigrants chez les catholiques. En outre, selon les résultats du recensement canadien - déjà ancien - de 1991, parmi les 1002945 descendants autochtones, inuits ou métisses canadiens d'Amérique du Nord, seulement 10840 ont déclaré avoir conservé les croyances traditionnelles de leur peuple.

\section{Religions ou spiritualités autochtones en émergence}

Les spiritualités autochtones sont difficiles à cerner avec précision. Le site du ministère des Affaires indiennes, tout comme les différents informateurs que nous avons pu rencontrer, autochtones ou non, ne parlent pas de "religion », mais de "spiritualité »: "Dans le discours autochtone, la spiritualité n'est pas un système de croyances qui se définit comme une religion; il s'agit d'un mode de vie selon lequel on reconnaît que chaque élément du monde matériel est en un sens imprégné de vie spirituelle et que tous les comportements humains sont influencés par une dimension immatérielle, sur laquelle ils agissent à leur tour ${ }^{16}$.» Bien que le phénomène n'ait pas encore été étudié de près, il semblerait qu'on assiste, d'après ce qui nous a été rapporté, à un regain important des spiritualités autochtones, qui sont de nouveau pratiquées par les membres les plus jeunes des communautés, mais ce renouveau prend volontairement place hors du cercle " officiel » des instances gouvernementales, et les différents anthropologues que nous avons rencontrés ont mentionné les réticences émanant des communautés autochtones dès lors qu'il s'agit de détailler certaines pratiques religieuses, ou ressortissant au sacré.

\section{Les religions juive et musulmane : une intégration conflictuelle?}

11 Les cas d'accommodements raisonnables concernant surtout les religions juive et musulmane, nous nous proposons ici de faire un très rapide état des lieux, tout en mentionnant quelques-uns des cas qui sont ressortis ces dernières années, liés plus ou moins directement au patrimoine religieux des communautés concernées. Les premières communautés judaïques remontent au XVIII siècle, et sont concentrées surtout dans la région de Montréal. Ces communautés ne sont pas homogènes, mais au contraire, d'origine diverse, ashkénazes au début du $\mathrm{XX}^{\mathrm{e}}$ siècle, puis sépharades francophones après la Seconde Guerre mondiale (actuellement, on estime que ces communautés sépharades francophones seraient composées d'environ 100000 pratiquants à Montréal). Deux cas liés au patrimoine religieux ont fait l'objet de l'intervention de différentes commissions dans le cadre des accommodements raisonnables. Les deux cas concernent la commune d'Outremont. Dans le premier cas, en 1990, la commune s'oppose à l'installation du érouv, sorte de clôture symbolique délimitant l'espace sacré dans lequel doit se dérouler le sabbat, par la communauté hassidique. La Cour supérieure a rendu son jugement en 2001, autorisant l'installation du érouv (en l'occurrence, dans ce cas précis, un fil de nylon érigé à environ 5 mètres de hauteur $)^{17}$. Dans le deuxième cas, des habitants d'Outremont se sont opposés en 1998 à l'édification de souccah par des juifs orthodoxes (les souccahs sont des tentes érigées à l'occasion de Soukkot ${ }^{18}$, commémorant la libération de l'esclavage en Égypte). Il s'agissait dans ce cas précis de tentes en tissu, érigées sur les balcons des 
appartements habités par la communauté juive. Cette interdiction a été confirmée en 2002 par la cour d'appel, mais rejetée en 2004 par la Cour suprême du Canada.

Les premières communautés musulmanes se sont installées à partir des années 1960, en provenance d'Égypte et du sous-continent indien, rejointes dans les années 1970 par les communautés originaires du Liban. La politique du Québec en faveur de la francophonie a favorisé à partir des années 1980 une immigration en provenance des pays du Maghreb. Il semblerait qu'actuellement, la concentration la plus forte de musulmans se situe à Montréal. Il faut noter que depuis ces cinq dernières années, on assiste parmi certains membres de la communauté musulmane à un deuxième mouvement migratoire : partis s'installer dans un premier temps au Canada francophone, certains choisissent ensuite de s'installer au Canada anglophone. Pour la religion musulmane, il semblerait que les problèmes liés aux pratiques religieuses aient surtout concerné la possibilité d'établir des lieux de prière. C'est le cas par exemple en 2003 des étudiants musulmans de l'École de technologie supérieure qui se voient refuser un lieu de prière par l'école, mais obtiennent finalement gain de cause en 2006 après avis de la commission des droits de la personne et des droits de la jeunesse. Une polémique a également surgi, sans qu'il y ait eu d'arbitrage demandé à une commission, lorsqu'en 2007, des musulmans ont prié après le repas de midi dans une cabane à sucre en Montérégie. Les cabanes à sucre sont des lieux hautement emblématiques de la sociabilité québécoise, car c'est là que se réunissent les communautés pour la confection du sirop d'érable. Les autres cas d'accommodements raisonnables liés à la religion musulmane ne portent pas directement sur des lieux, patrimoniaux ou non, dans lesquels les musulmans auraient souhaité prier, ou sur des objets du culte, mais sur les signes visibles de l'appartenance religieuse, comme le port du voile, ou bien encore, sur la séparation entre les sexes lors d'activités sportives.

\section{Que faire des bâtiments religieux ?}




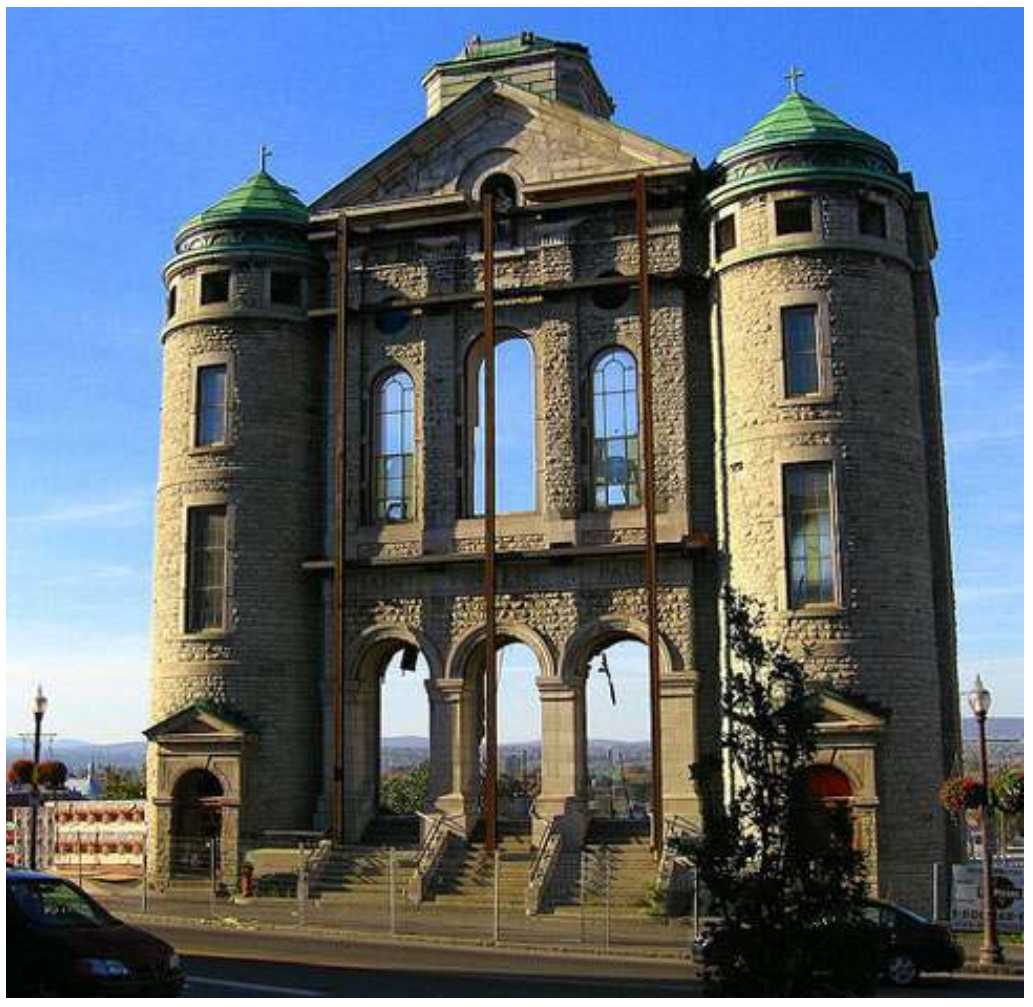

Façade de l'église Saint-Vincent-de-Paul de Québec

Photo Louise Saint-Pierre (c) Louise Saint-Pierre

Des religions ancrées depuis longtemps, comme le catholicisme et le protestantisme, disposent d'un patrimoine bâti, mobilier et immobilier, important, surtout pour la religion catholique. Dans ce cas se pose la question de savoir comment gérer le passage entre la baisse de la pratique, et l'utilisation nouvelle à donner au patrimoine matériel. Pour la religion catholique en particulier, la baisse du nombre des pratiquants et des membres du clergé s'accompagne d'une baisse de la fréquentation des églises, qui entraine leur fermeture, leur transformation, ou leur destruction. Luc Noppen et Lucie K. Morisset estiment que durant cette période, 135 paroisses ont été supprimées, et que $25 \%$ (soit 453) du total des églises et chapelles catholiques ont été désaffectées ${ }^{19}$. Face à cette situation récente, se pose le problème de l'utilisation et/ou du devenir des bâtiments. Le cas-repoussoir est, à Québec même, l'église Saint-Vincent-de-Paul, sur la côte d'Abraham, à l'entrée du centre-ville historique : il n'en reste plus aujourd'hui que la façade, le reste ayant été détruit par un promoteur, sans autorisation préalable, dans l'espoir de construire un hypothétique hôtel de tourisme ${ }^{20}$. (fig. $\mathbf{n}^{\circ}$ 4) Ce cas est extrême, et traduit plus semble-t-il des difficultés de coordination entre les différentes parties responsables de la valorisation du bâtiment. Il faut en effet noter que des mesures ont été prises ces dix dernières années pour sauvegarder ce patrimoine. Par exemple, en 1999, une déclaration conjointe a été signée entre l'Archevêché de Québec, la Ville de Québec et le ministère de la Culture et des Communications « concernant la sauvegarde et la mise en valeur des églises situées sur le territoire de l'ancienne Ville de Québec ». 
Figure 5

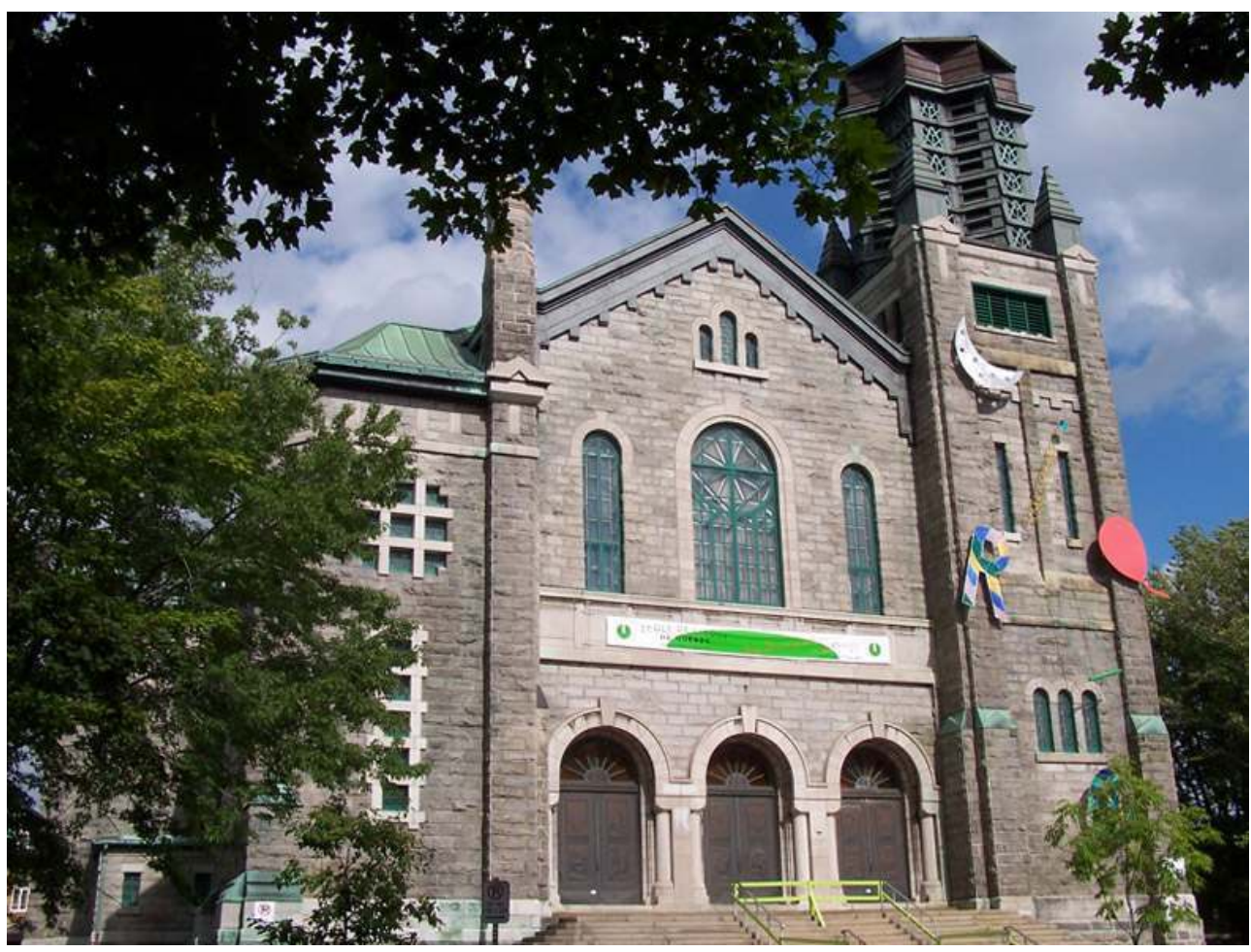

Ancienne église Saint-Esprit de Québec, devenue l'École du cirque de Québec. Façade

(c) François Bastien

14 C'est dans ce cadre que s'est opérée la rénovation de l'église Saint-Esprit, dans le quartier de Limoilou à Québec, construite en 1941, désaffectée, et qui abrite depuis 2002 l'École de cirque de Québec. L'intérieur de l'église a été réaménagé pour permettre aux élèves de pratiquer leur art, tout en respectant l'esprit du lieu. Cette réalisation, due au cabinet $A B C P$ architecture et urbanisme, a été saluée de manière unanime. Le commentaire de LouisGuy Lemieux, journaliste au Soleil, paru sur le site internet Québec urbain, dans la rubrique " coups de cœur urbains », résume bien la nature de la contribution du cabinet, qui a réussi à vaincre les réticences face à la transition a priori difficile entre un lieu de culte et un lieu dédié à une pratique à la fois ludique, artistique et sportive, tout en rappelant les enjeux liés à la conversion des églises : « Il s'agit du recyclage de l'ancienne église SaintEsprit de Limoilou en école de cirque. À première vue, cette fonction semble incompatible avec le caractère sacré d'un lieu de culte, mais cette reconversion s'est finalement avérée un choix judicieux, en raison du grand espace et surtout de la grande hauteur qu'offrait l'intérieur de l'église [note: la nef fait $15 \mathrm{~m}$ de hauteur, $24 \mathrm{~m}$ de largeur et $60 \mathrm{~m}$ de profondeur]. Tout en intégrant des éléments contemporains aux couleurs ludiques, les concepteurs ont fait preuve d'une grande sensibilité quant au respect de l'architecture existante, en s'assurant de la réversibilité des interventions. [Advenant] le départ de l'école de cirque, l'église pourrait reprendre son aspect d'origine ou être reconvertie à d'autres fins. J'ai choisi cette réalisation car elle ouvre la porte à l'inventivité et à la créativité dans le recyclage des lieux de culte, principal défi de conservation architecturale dans les prochaines années, étant donné la grande quantité d'églises qui seront disponibles et la difficulté de leur trouver de nouvelles vocations compatibles ${ }^{21}$. » 


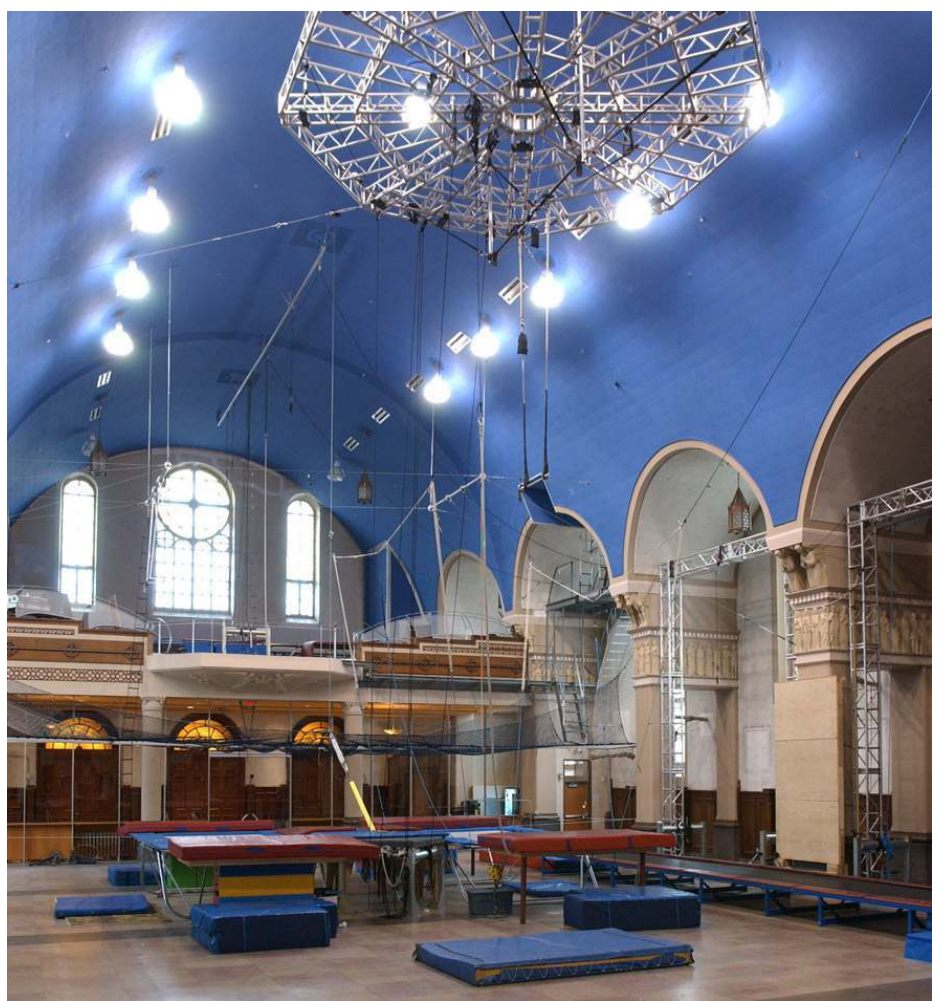

École du cirque de Québec. Intérieur

(c) François Bastien

La négociation traditionnelle de l'Occident, qui répond à la baisse des pratiques par la patrimonialisation, peut jouer à plein dans les cas mentionnés ci-dessus, comme le souligne Laurier Turgeon, pour qui le problème de la « conversion de ces biens d'Église à vocation essentiellement cultuels en des biens culturels destinés à la société civile» s'opère par une patrimonialisation, dont la fonction est de "sauvegarder cet héritage ${ }^{22}$ ". De l'autre côté, perdurent, voire se ré-affirment des spiritualités autochtones qui utilisent comme supports de leurs pratiques des structures architecturales souvent éphémères, en même temps que les communautés immigrées font entendre leur voix, alors qu'elles ne disposent pas d'un patrimoine bâti encore totalement visible, ou bien doivent lutter pour affirmer la visibilité de leurs pratiques.

\section{Définir, inventorier, transmettre, ou la définition d'une politique patrimoniale}

Face à cette hétérogénéité, la réponse de l'État canadien passe d'abord par l'établissement de définitions, qui posent des bornes délimitant l'action des diverses administrations. On l'a vu plus haut, le ministère des Affaires indiennes a donné une définition de ce qu'elle entend par «spiritualités autochtones ». De même, la commission des droits de la personne et des droits de la jeunesse a-t-elle donné une définition de ce qu'elle entend par religion : « Le concept de la religion désigne un ensemble de doctrines et de pratiques ayant pour objet les rapports de l'âme humaine avec le sacré et en fonction duquel une communauté de croyants partage certains sacrements, rites ou un code moral ${ }^{23}$.» Cette 
définition relie la pratique religieuse à la fois à l'individu, qui vit un rapport particulier au sacré, et à la communauté, qui est en charge des manifestations extérieures de la foi. La séparation entre pratique intérieure, propre à chaque individu dans son espace privé, et pratique extérieure, liée à la communauté, permet à l'État québécois d'agir sur ce qui ressortit au groupe, et aux pratiques que celui-ci déploie dans l'espace public.

\section{« Laïcité-dialogue » et patrimoine religieux}

Les rapports entre religion, spiritualités et patrimoine sont précisés plus avant dans un document, paru en 2006, intitulé Croire au patrimoine, et qui peut être considéré comme un des principaux actes fondateurs de la politique contemporaine de l'État en matière de patrimoine religieux ${ }^{24}$. Il a été rédigé par les membres de la commission culture du Québec, et publié par l'Assemblée nationale du Québec.

18 L'intervention de l'État sur le religieux est régie par le concept, central au rapport, de "laïcité-collaboration, voire laïcité-dialogue ", défini comme "celle qui reconnaît la présence du phénomène religieux dans la société civile et favorise son épanouissement ». Cette conception d'un État laïque veillant à une séparation entre le laïque et le religieux, tout en favorisant la collaboration et le dialogue entre les deux, est entourée dans le rapport d'un recours parfois insistant à l'ensemble des dispositifs législatifs qui justifient dans le droit, selon les auteurs, la légitimité de cette conception. L'introduction cite ainsi toute une série de textes qui sont invoqués pour corroborer la position de la commission : charte des droits et libertés de la personne, qui établit que « toute personne est titulaire des libertés fondamentales telles [...] la liberté de religion» et que «toute personne a droit à la reconnaissance et à l'exercice, en pleine égalité, des droits et libertés de la personne, sans distinction, exclusion ou préférence fondée sur [...] la religion »; loi sur la liberté des cultes qui prévoit, quant à elle, que « la jouissance et le libre exercice du culte de toute profession religieuse, sans distinction ni préférence, mais de manière à ne pas servir d'excuse à la licence, ni à autoriser des pratiques incompatibles avec la paix et la sûreté au Québec, sont permis par la constitution et les lois du Québec à toutes les personnes qui y vivent»; et pacte international relatif aux droits civils et politiques auquel le Canada a l'obligation de se conformer. Ces différents dispositifs forment un cadre qui permet à la commission d'établir que la société québécoise est fondée sur « un modèle de laïcité auquel les membres de la Commission adhèrent et qu'il serait opportun de maintenir dans un Québec soucieux de sa diversité religieuse ».

\section{Inventorier pour connaître, puis choisir}

Le rapport détaille ensuite quatre grands mouvements nécessaires à l'élaboration d'une politique en matière de patrimoine religieux : connaissance, protection, transmission et gestion. Attardons-nous plus précisément sur la notion de connaissance, car elle soulève un point intéressant : la connaissance est ici envisagée sous l'angle, non pas d'études de type universitaire, mais comme devant se matérialiser par un inventaire, qui permettra ensuite aux autorités publiques d'effectuer des choix "puisque pour faire des choix éclairés, on doit connaitre l'objet sur lequel on se prononce. Dans le cas du patrimoine religieux, force est de constater que notre connaissance est fragmentaire et qu'il faut compléter les inventaires des biens religieux immobiliers, confectionner des inventaires des biens mobiliers et instituer un programme d'enquêtes destiné à faire connaître le 
patrimoine religieux immatériel ». De ce besoin de connaissance, le rapport tire deux recommandations: "Recommandation $\mathrm{n}^{\circ} 1$ : La Commission recommande que le ministère de la Culture et des Communications prépare, dans les plus brefs délais, une liste des inventaires, terminés ou non, et constitue un répertoire de tous les inventaires consacrés au patrimoine religieux matériel et que ce répertoire soit mis à jour annuellement » et "Recommandation $n^{\circ} 2:$ La Commission recommande qu'à partir de cette liste des inventaires, le nouveau conseil du patrimoine religieux du Québec détermine les secteurs où des inventaires doivent être effectués en priorité, qu'il définisse une méthodologie reconnue et qu'il étudie la possibilité de mettre en place un outil de recherche réunissant les données de tous ces inventaires. » L'inventaire est donc conçu à la fois comme un outil de connaissance, mais aussi comme un moyen de sélectionner, de choisir ce qui pourra être digne de devenir patrimoine.

\section{Le rôle du patrimoine immatériel}

20 Autre point important du rapport, la première reconnaissance, dans un rapport officiel, de la notion de patrimoine immatériel : « La Commission est d'avis qu'il y a lieu de dresser un inventaire du patrimoine religieux immatériel avec la participation des communautés et les groupes qui créent, entretiennent et transmettent ce patrimoine ", pour dresser un «inventaire des paroles et des gestes au Québec.» Cependant, il s'agit surtout d'un récolement par des moyens audiovisuels de paroles de témoins sur des faits du passé. Le rapport cite ainsi un extrait d'une communication de Diane Audy, ethnologue spécialiste du domaine religieux, où la définition du patrimoine immatériel ressortit à celle de récolement de témoignages oraux sur des pratiques disparues, tout empreinte d'un sentiment d'urgence : «La moyenne d'âge actuelle des principaux acteurs est de 80 ans. (...) Il faut donc, de toute urgence, prendre les dispositions nécessaires afin de recueillir les témoignages de ceux et celles qui ont bâti et utilisé les lieux de culte (...) Le délai est très court; il ne reste que cinq ans, dix ans au maximum pour obtenir des témoignages valables. Il n'y a pas de relève. » Or, si l'on se réfère à la définition du patrimoine culturel immatériel donnée par ailleurs par la Convention de l'Unesco de 2003 sur la sauvegarde du patrimoine culturel immatériel, celui-ci n'est pas envisagé en termes de ruptures possibles, mais au contraire, de continuité historique: "Ce patrimoine culturel immatériel, transmis de génération en génération, est recréé en permanence par les communautés et groupes en fonction de leur milieu, de leur interaction avec la nature et de leur histoire, et leur procure un sentiment d'identité et de continuitée ${ }^{25}$." Si l'on appliquait à la lettre la définition de l'Unesco, on pourrait dire qu'en l'occurrence, il n'y a pas - ou plus - de patrimoine immatériel dans ce que les auteurs du rapport choisissent de valoriser...

\section{L'inventaire du patrimoine immatériel religieux au Québec}

Suite à ce rapport, et à l'insistance portée sur le patrimoine immatériel religieux, un inventaire du patrimoine religieux a été entrepris sous l'égide du gouvernement, en collaboration avec les instances universitaires. L'université Laval a été chargée de travailler à l'inventaire du patrimoine immatériel religieux (IPIR), dans la foulée du travail qu'elle a déjà accompli sur l'inventaire du patrimoine immatériel au Québec (IREPI). Il devrait pouvoir bénéficier d'une somme de 600000 dollars canadiens sur trois ans. Le texte qui suit s'appuie largement sur le rapport de pré-enquête, non encore 
publié, que les responsables de cet inventaire, Laurier Turgeon et Louise Saint-Pierre ${ }^{26}$, ont rendu au ministère de la Culture en $2008^{27}$.

Ce rapport donne une définition intéressante du patrimoine immatériel religieux ${ }^{28}$. Les auteurs le placent d'emblée sous l'angle du culturel, et ne mentionnent à aucun moment la notion de laïcité-ouverte: "Nous avons adopté une définition du patrimoine immatériel religieux assez large qui déborde le caractère strictement religieux et spirituel pour atteindre la vie culturelle et la mémoire des religieuses et des religieux. Il faut d'abord dire que limiter le patrimoine religieux à la seule sphère du sacré serait très restrictif et nous ferait passer à côté de l'essentiel qui est d'ordre culturel ${ }^{29}$. On peut voir là le début d'une opposition, et, peut-être, d'une fracture possible, entre ce que les scientifiques et le ministère de la Culture entendent analyser comme des phénomènes à étudier en tant qu'objets de culture, et ce que les pratiquants estiment être des traces de leurs pratiques. Cette distinction est apparue assez fortement lors d'un colloque consacré à la question du patrimoine religieux auquel nous avons assisté en mai 2008. L'allocution d'ouverture était prononcée par un psychiatre catholique qui a insisté sur le fait que l'immatériel, pour un catholique pratiquant, signifiait non seulement la description des gestes et des pratiques, mais aussi les résonances personnelles que le culte pouvait engendrer : "Le plus souvent, le patrimoine religieux est perçu à partir de son côté matériel et lorsqu'on se réfère au patrimoine immatériel religieux, on a tendance à se limiter aux facettes susceptibles de devenir plus observables à partir des narratifs obtenus (mode de vie, savoir-faire, comportements divers). Ceci limite l'observation et ne réfère pas au noyau essentiel de l'expérience religieuse qui est celui d'une vie avec des caractéristiques particulières [...] c'est en ce sens qu'il faudra trouver les moyens de référer à l'identité propre du religieux, non pas en s'y limitant dans sa spécificité ultime, mais en intégrant constamment celle-ci à l'intérieur de toute forme de témoignage ou de tout lien avec les artefacts dont on expliquerait le sens ${ }^{30}$."

Un autre point important du rapport concerne la nécessité d'opérer des choix et non de prétendre à l'exhaustivité : «Une définition large de ce patrimoine ne signifie pas qu'il faut tout inventorier. Au contraire, il est important d'être sélectif, en ne retenant que ses éléments les plus représentatifs. La convention de l'Unesco sur la sauvegarde du patrimoine culturel immatériel reconnaît la représentativité comme un élément fondamental du patrimoine immatériel : « "Représentatif » pourrait signifier, à la fois, représentatif de la créativité de l'homme, du patrimoine culturel des États, mais aussi du patrimoine culturel des communautés qui sont les détenteurs des traditions en question ${ }^{31}$ . » Le patrimoine est par définition exclusif; il doit demeurer dans l'ordre du singulier et de l'exceptionnel pour conserver sa valeur. Faire de tout l'immatériel religieux du patrimoine ne contribuera qu'à le dévaluer.

Le déroulement du pré-terrain est ensuite détaillé, et nous avons pensé qu'il était important de s'y attarder, car il permet d'étudier la manière dont les principes établis à la fois par le ministère et l'université ont pu être déclinés de manière pratique. D'abord, le projet englobe à la fois la religion catholique et les religions issues de la diversité des pratiques et des origines : les instigateurs du projet-pilote ont sélectionné à la fois des communautés catholiques et des communautés appartenant à d'autres confessions. La raison invoquée, là encore, n'est pas de l'ordre de l'expression de la "laïcité-ouverte ", mais de l'ordre du culturel : « Le nouveau programme adopte une approche culturelle du phénomène religieux : il permet de poser un regard sur le patrimoine religieux du Québec dans toutes ses dimensions (immobilier, mobilier et immatériel). L'accent sera mis sur les 
traditions religieuses qui ont marqué le Québec (catholicisme, protestantisme, judaïsme et spiritualité autochtone) mais s'intéressera également aux traditions religieuses anciennes (bouddhisme, islam, hindouisme, etc.) implantées plus récemment au Québec ${ }^{32}$ . » Ensuite apparaît très clairement l'idée qu'il s'agit de choix effectués sciemment, sur des critères précis : les communautés « ont été choisies en fonction de leur ancienneté, de leur patrimoine et de leur dynamisme ${ }^{33}$ ». Le projet pilote a sélectionné trois congrégations catholiques, les Augustines et les Ursulines de Québec, et les Sulpiciens de Montréal, en raison de leur importance numérique, et du poids important qu'elles ont joué dans l'histoire du Québec. Ont également été sélectionnées la cathédrale anglicane Holy Trinity de Québec, la communauté francophone protestante de l'Église évangélique baptiste de Marieville, la communauté juive rassemblée autour de la Spanish and Portuguese Synagogue de Montréal, la plus ancienne du Canada, la communauté orthodoxe de la paroisse Saint-Pierre-et-Saint-Paul et la communauté de Rawdon, et enfin la communauté amérindienne des Innus de la Côte-Nord.

Pour finir, le rapport distingue quatre types de "récits », qui structurent l'inventaire : « récits de lieu qui portent sur l'usage et le sens des espaces les plus significatifs dans chacune des communautés, les hauts lieux de l'habitat. Ex. : chapelle, sacristie, jardin, grotte, réfectoire, salle d'enseignement, cimetière, presbytère, synagogue, lieux de culte, espaces communautaires [...] ; récits d'objet qui renvoient aux objets matériels ayant une valeur symbolique. Ex.: objets religieux, vêtements liturgiques, habits traditionnels, mobilier traditionnel, mobilier de cuisine, etc. [...], récits de vie qui visent à documenter des pratiques reliées à la vie communautaire, spirituelle et professionnelle renfermant un caractère plutôt exceptionnel et donc une valeur patrimoniale [...] et récits de pratiques cultuelles et culturelles qui regroupent les dévotions particulières, les coutumes funéraires, les pratiques liturgiques significatives, les pratiques professionnelles marquantes, les savoir-faire uniques ayant une valeur à la fois pragmatique et symbolique dans la communauté (la statuaire, la broderie, la dentellerie, la dorure, le tressage, la fabrication d'objets religieux, le reprisage, la fabrication de produits alimentaires, etc. $)^{34}$ . \)

Les résultats de ce travail ont vocation à être mis en ligne sur un site internet, qui devrait voir le jour à l'été 2009 , et qui lierait patrimoines matériel et immatériel religieux, en partant du travail de l'IPIR pour l'immatériel, et de travaux déjà effectués par ailleurs pour l'inventaire du patrimoine matériel religieux ${ }^{35}$. Comme on peut le voir dans le fichier joint, l'idée de ce site est de partir du matériel (dans le cas montré ici, une église), pour y adjoindre des éléments d'information de l'ordre des pratiques et du savoir-faire (dans ce cas, l'art de sonner les cloches). L'immatériel y est adjacent à l'objet, qui reste le point d'ancrage premier pour le public. L'immatériel est envisagé sous la forme de récits, avec deux dimensions, que nous appellerons celle du passé simple, et celle du présent absolu, tant le recours à l'analyse grammaticale peut se révéler pertinent dans ce cas. Le passé simple est le temps de la narration, de l'expression successive d'événements qui sont achevés. Les récits de vie, comme celui du prêtre sulpicien interrogé sur son action d'évangélisation au Japon, participent de cette valeur temporelle de l'achevé, du nonreproductible. Le présent absolu est utilisé pour exprimer des actions qui se reproduisent constamment, ou qui sont toujours vraies. Il désigne des actions de l'ordre du générique, comme pour le récit du prêtre à qui l'enquêteur demande de présenter un ostensoir, où l'informateur n'est pas sollicité sur les usages précis de l'ostensoir de son église. Ce présent absolu de l'immatériel est le fruit d'une volonté du gouvernement, comme il est 
expressément écrit dans la recommandation 18 du rapport Croire au patrimoine: "Le ministère de l'Éducation s'assure qu'un volet de sensibilisation au patrimoine religieux soit inclus dans le nouveau programme d'éthique et de culture religieuse qui est en cours d'élaboration: construction d'un patrimoine par, d'abord, sa mise en abstraction, son apurement de ses spécificités contextuelles. » L'informateur, par la nature des « récits » qu'il est convié à raconter, devient une sorte de pédagogue décrivant des rites dans l'absolu, et non les pratiques spécifiques à chaque milieu et à chaque groupe.

Pour connaître, l'État a besoin d'inventorier, et pour renforcer encore une approche cohérente, fait appel au patrimoine immatériel, car s'il n'existe pas toujours de traces matérielles des pratiques, il reste toujours des récits, des mémoires et des gestes, quel que soit le degré de profondeur historique des communautés. Confronté à une situation protéiforme, et dans son souci d'affirmer le principe de laïcité-dialogue, l'État québécois a donc ainsi l'ambition de traiter des diversités religieuses de manière homogène. Partant, ce parti pris renforce l'idée de communautés cohérentes, aux contours bien définis, agissant au sein d'un espace public laïc, sous l'égide de l'État, et donne une vision unifiée de groupes qui ne le sont sans doute pas tant.

Puisque nous avons commencé ce texte par un débat, terminons-le par une histoire. Cette fois-ci, il s'agit d'un film de Pierre Perrault, figure tutélaire des documentaristes québécois, qui décrit les pratiques de la pêche au marsouin sur l'Isle-aux-Coudres, près de Québec, dans les années 1960. L'auteur demande à ses habitants de recréer à nouveau cette pêche, disparue depuis une quarantaine d'années. Les hommes sont face à la nuit, rassemblés au bord de la mer, puis se mettent à parler de la création de la lune. Par la magie de la caméra, dans ce bout du monde perdu au milieu d'un autre monde, anglophone, protestant, ressurgit l'âme du Québec, empreinte du vieux catholicisme de l'Ancien Monde, et de son paganisme aussi. L'âme, ou une partie de l'âme? Car si l'identité des Québécois se fonde en bonne partie sur la mémoire des (im)migrations qu'ils ont eux-mêmes entreprises à partir du XVIe siècle, ils doivent désormais composer avec d'autres mouvements d'immigration, nécessaires au développement économique et démographique de la Province, qui n'amènent plus seulement des personnes originaires d'Europe, mais d'autres pays, d'autres cultures, d'autres religions. Et il est piquant d'imaginer qu'au moment même où Perrault plantait sa caméra, à une centaine de kilomètres de là, à Montréal, s'installaient les premières communautés musulmanes. Le film de Perrault s'intitule Pour la suite du monde et l'on peut se demander, au regard de l'histoire contemporaine, des interrogations posées par la notion de patrimoine religieux au Québec, de quelle suite il s'agira : celle d'un monde dont les croyances, les pratiques, les gestes, sont en train de mourir, qu'il faut préserver, ou celle d'un autre monde, complexe, hétérogène, porteur de crises, où les rencontres entre cultuel et culturel seront une des clés pour tenter de cerner enfin une identité qui peine à s'affirmer? 


\section{BIBLIOGRAPHIE}

Coomans, Thomas, Morisset, Lucie K., Noppen, Luc (dir.) [traduction, Daly-Dallaire ... et al.]. Quel avenir pour quelles églises? What future for which churches? Québec ; Presses de l'Université du Québec, 2006.

Dumont, Fernand. Genèse de la société québécoise. Montréal : Les éditions du Boréal, 1996.

Noppen, Luc et Morisset, Lucie K. Les églises du Québec : un patrimoine à réinventer. SainteFoy : Presses de l'Université du Québec, 2005.

Rocher, Marie-Claude et Pelchat, Marc (dir.) Le patrimoine des minorités religieuses au Québec, richesse et vulnérabilité. Québec : Presses de l’Université Laval, 2006.

Simard, Jean. Le patrimoine religieux au Québec : exposé de la situation et orientations. Rapport réalisé par la Commission des biens culturels. Québec : publications du Québec, 1998.

Turgeon, Laurier (dir.) Le patrimoine religieux du Québec : entre le cultuel et le culturel. Québec : Presses de l'Université Laval, 2005.

\section{NOTES}

1. Voir sur le site : http://fr.wikipedia.org/wiki/Accommodement_raisonnable.

2. Nonobstant les articles parus dans des périodiques volontiers polémiques, le lecteur peut consulter le dossier interactif mis en ligne sur Radio Canada. Voir sur le site : http://www.radiocanada.ca/nouvelles/societe/2007/01/10/011-laicite-quebec2.shtml. Il est intéressant de noter que les photographies mises en ligne avec ce rapport mettent en avant des pratiques qui sont le plus susceptibles de heurter les mentalités des Québécois « de souche ». Elles montrent en effet des jeunes filles musulmanes voilées dans une classe, et un représentant de la communauté sikh portant turban et barbe, alors que le port du voile, ou des signes religieux sikhs, n'ont été qu'une part des cas soumis à la procédure des accommodements raisonnables. Un rapide sondage dans les différents forums de discussion québécois consacrés à ce sujet n'a pas fait apparaitre de débats particulièrement nourris sur la question. A chaque fois, sur les forums consultés en avril 2009, la discussion n'a attiré que deux ou trois réponses. Nous n'avons pas trouvé non plus beaucoup de blogs directement polémiques sur la question.

3. Charles Taylor est professeur émérite de philosophie et de science politique à l'université McGill de Montréal. Il a beaucoup travaillé sur les notions de multiculturalisme (voir Multiculturalism and the Politics of Recognition, paru en 1992 aux presses de l'université de Princeton, traduit en français et édité par Flammarion en 1997 sous le titre Multiculturalisme). Il a également publié en 1992 un essai intitulé Rapprocher les solitudes. Écrits sur le fédéralisme et le nationalisme au Canada, Québec, Presses de l'Université Laval.

4. Gérard Bouchard est professeur de sociologie à l'université du Québec à Chicoutimi. Il a publié des ouvrages sur la nation et la culture québécoise, comme La nation québécoise au futur et au passé, 1999 ou La culture québécoise est-elle en crise ?, 2007.

5. Voir le site: http://www.accommodements.qc.ca/commission/mandat.html qui fournit le texte du mandat accordé aux deux auteurs du rapport. 
6. Bouchard, Gérard, Taylor, Charles. Fonder l'avenir. Le temps de la conciliation, 310 pages. Le rapport complet est disponible sur le site: http://www.accommodements.qc.ca/ documentation/rapports/rapport-final-integral-fr.pdf.

7. Bouchard, Gérard, Taylor, Charles. Fonder l'avenir. Le temps de la conciliation, p. 20.

8. Voir sur le site: http://www.radio-canada.ca/nouvelles/National/2008/05/22/003-reax-BTpolitique.shtml>.

9. Voir sur le site: http://www.radio-canada.ca/nouvelles/National/2008/05/22/003-reax-BTpolitique.shtml>.

10. Cette mission a été effectuée dans le cadre de la convention de coopération culturelle entre les ministères français et québécois de la Culture. Son but était de mettre en place des échanges relatifs à l'inventaire du patrimoine culturel immatériel en France et au Québec. Je tiens à remercier le ministère québécois de la Culture et l'université Laval pour leur accueil, et tout particulièrement Daniel Lauzon et Karine Laviolette, du ministère de la culture, ainsi que Laurier Turgeon, Martine Roberge, Louise St Pierre et Célia Forget de l'université Laval. Sans leur aide et leur soutien, cet article n'aurait jamais pu être rédigé.

11. Dumont, Fernand. Genèse de la société québécoise, p. 9-10.

12. Simard, Jean. Le patrimoine religieux au Québec : exposé de la situation et orientations. Rapport réalisé par la Commission des biens culturels. Québec: publications du Québec, 1998, p. 18.

13. Simard, Jean. Le patrimoine religieux au Québec : exposé de la situation et orientations. Rapport réalisé par la Commission des biens culturels. Québec: publications du Québec, 1998, p. 74.

14. Le Québec doit faire face à une immigration importante, qu'il a lui-même favorisée. Sur une population de 7 millions d'habitants, environ 363500 immigrants ont été admis entre 1997 et 2006, dont près de $90 \%$ sont restés sur place. [Bulletin statistique du premier trimestre $2008 \mathrm{du}$ ministère de l'Immigration et des communautés culturelles du Québec ; voir sur le site : http:// www.micc.gouv.qc.ca/fr/recherches-statistiques/stats-etablis-immigrants.html>] De 2003 à 2007, les pourcentages par continent d'origine s'élèvent à 29,9\% pour l'Asie, 27,5\% pour l'Afrique (dont 18,2 \% pour l'Afrique du Nord), 22,2 \% pour l'Europe et 20,2 \% pour l'Amérique. [Tableaux sur l'immigration du Québec 2003-2007, mars 2008; voir sur le site: http:// www.micc.gouv.qc.ca/publications/fr/recherches-statistiques/Immigration_Qc_2003-2007.pdf>.

15. Statistique Canada, Recensement de 2001: série "analyses", Les religions au Canada, catalogue $n^{\circ}$ 96F0030XIF2001015, 13 mai 2003.

16. Site du ministère des Affaires indiennes et du Nord Canada, $3^{e}$ partie, chapitre $15 \mathrm{du}$ rapport de la commission royale sur les peuples autochtones, «Ranimer la flamme »; voir sur le site : http://www.ainc-inac.gc.ca/ch/rcap/sg/sg52_f.html.

17. Une photo du érouv est disponible sur le site: http://lcn.canoe.ca/infos/regional/ archives/2001/06/20010621-121540.html.

18. Soukkot, ou "fête des tentes" (hag ha-Soukkot) commémore l'errance des Juifs dans le désert. Suivant un passage du Lévitique («Vous habiterez sept jours sous des huttes (Soukkot). Tous les citoyens d'Israël habiteront sous des huttes, afin que vos descendants sachent que j'ai fait habiter sous des huttes les Israélites quand je les ai fait sortir du pays d'Égypte. Je suis le Seigneur votre Dieu. » Lv 23, 42-43), les Juifs érigent une tente à leur domicile, dans leur jardin ou sur leur balcon, et doivent y prendre tous leurs repas. Cette fête dure une semaine au début de l'automne.

19. Noppen, Luc et Morisset, Lucie K. Les églises du Québec : un patrimoine à réinventer. Sainte-Foy : Presses de l'Université du Québec, 2005, p 1.

20. Cette destruction, et l'état dans lequel cette église a été laissé, a soulevé de nombreuses polémiques à Québec. Voir à ce sujet un article de Québec Hebdo sur le site: http:// www.quebechebdo.com/article-202844-La-facade-de-leglise-SaintVincentdePaul-est- 
sauvee.htm>l. Pour un écho des polémiques, on peut également consulter le forum consacré à l'église sur le site de Québec urbain, dédié à l'urbanisme de Québec, en collaboration avec la commission de la capitale nationale de Québec sur le site: http://quebecurbain.qc.ca/forum/ index.php ?showtopic =1326).

21. Voir sur le site : http://www.quebecurbain.qc.ca/2004/08/02/coups-de-coeur-urbains/. Voir également l'article de Luc Noppen et Lucie K. Morisset dans la revue ARQ de mai 2005, consacrée à la reconversion des églises du Québec. Cette réalisation a remporté le Prix du public et le Prix du jury dans la catégorie « recyclage » des Mérites d'architecture de la Ville de Québec.

22. Simard, Jean. Le patrimoine religieux au Québec : exposé de la situation et orientations. Rapport réalisé par la Commission des biens culturels. Québec: publications du Québec, 1998, p. 19-20.

23. Eid, Paul et Avila, Ramon. La place de la religion dans l'espace public, Commission des droits de la personne et des droits de la jeunesse, Québec, décembre 2006. Voir sur le site : http:// www.cdpdj.qc.ca/fr/placedelareligion/docs/religion-Quebec-statistiques.pdf>

24. Un résumé du rapport est disponible sur http://www.revparl.ca/francais/issue.asp? art $=1201 \&$ param $=178 "$.

25. Voir sur le site: http://www.unesco.org/culture/ich/index.php ?pg =00006, article 2 de la convention.

26. Je remercie Louise Saint-Pierre pour l'envoi d'illustrations pour cet article.

27. Projet pilote de l'Inventaire du patrimoine immatériel religieux. Bilan des activités. Rapport présenté au ministère de la Culture, des Communications et de la Condition féminine par Laurier Turgeon et Louise Saint-Pierre, Université Laval. Ce rapport inédit m’a été envoyé par messagerie en deux parties. La pagination indiquée est celle des documents qui m'ont été envoyés, et ne correspond peut-être pas à la pagination finale, telle qu'elle pourra apparaître dans le rapport imprimé soumis au ministère de la Culture québécois.

28. Voir aussi l'article de Florence Descamps dans cette livraison: Mémoire religieuse, patrimoine immatériel du religieux. Pour la constitution d'archives orales de la foi catholique au $\mathrm{XX}^{\mathrm{e}}$ siècle.

29. Projet pilote de l'Inventaire du patrimoine immatériel religieux. Bilan des activités, première partie, p. 8.

30. Conférence d'ouverture donnée par le docteur Harry Graham, professeur émérite de psychiatrie à la faculté de médecine de l'université Laval, lors du $13^{\mathrm{e}}$ colloque de la Mission Patrimoine religieux, 23 mai 2008.

31. Projet pilote de l'Inventaire du patrimoine immatériel religieux. Bilan des activités, p. 9.

32. Projet pilote de l'Inventaire du patrimoine immatériel religieux. Bilan des activités, deuxième partie, p. 1.

33. Projet pilote de l'Inventaire du patrimoine immatériel religieux. Bilan des activités, deuxième partie, p. 11.

34. Projet pilote de l'Inventaire du patrimoine immatériel religieux. Bilan des activités, deuxième partie, p. 19-20.

35. Voir Daniel Lauzon, «La base de données Patrimoine immobilier, mobilier et immatériel du Québec et le Répertoire du patrimoine culturel du Québec. État des lieux et perspectives pour l'inventaire du patrimoine immatériel ", actes du colloque Le patrimoine culturel immatériel de l'Europe. Inventer son inventaire. Voir sur le site :

http://mediatheque-numerique.inp.fr/index.php/actes_de_colloque/

rencontres_du_patrimoine/

le_patrimoine_culturel_immateriel_de_l_europe_inventer_son_inventaire 


\section{RÉSUMÉS}

Le but de cet article est de fournir quelques pistes d'analyse sur la question religieuse au Québec, en étudiant les oscillations constantes entre histoire, patrimonialisation et reconnaissance identitaire, qui traversent cette partie francophone du Canada. Nous tenterons de rendre compte de la manière dont est en train de se définir le patrimoine religieux au Québec, en étudiant le rôle des administrations culturelles, et tout particulièrement du ministère de la Culture québécois. Après un survol rapide de la situation des religions au Québec, cet article abordera ensuite plus précisément les mesures mises en place par le ministère de la Culture québécois pour identifier et faire connaître le patrimoine religieux, tout particulièrement immatériel, de la province.

The aim of this article is to display some elements that will help analyzing the religious issue in Quebec, through a study of the lasting interactions between history, heritage building and identity recognition. It will try to assess the way religious heritage is being defined in Quebec, through the study of the role of cultural administrations, more particularly the Ministry of Culture in Québec. After a quick survey of the situation of the various religions in Quebec, it will study more precisely the measures put forward by the Quebec Ministry of Culture in order to identify and let religious heritage of the province (and more particularly its intangible aspects) be more widely known.

\section{INDEX}

Mots-clés : laïcité-dialogue, protestantisme, judaïsme, spiritualités autochtones, pratiques cultuelles, patrimoine religieux, patrimoine immatériel, accommodements raisonnables, Révolution tranquille, inventaire, religion, Québec, Islam, catholicisme

\section{AUTEUR}

\section{SYLVIE GRENET}

Chargée de mission pour le patrimoine ethnologique, Direction de l'architecture et $\mathrm{du}$ patrimoine, ministère de la Culture et de la Communication. sylvie.grenet@culture.gouv.fr 